Journal of Management and Economics for Iberoamerica

Artículo de revisión

\title{
Responsabilidad Social Universitaria: una revisión sistemática y análisis bibliométrico
}

Pedro Duque *

Profesor Investigador, Facultad de Ciencias Administrativas, Económicas y Contables, Universidad Católica Luis Amigó, Manizales, Colombia.

Estudiante, Doctorado en Administración, Universidad Nacional de Colombia, Manizales, Colombia.

pedro.duquehu@amigo.edu.co

Luis-Salvador Cervantes-Cervantes

Director Académico, Universidad del Valle de Atemajac, Zapopan, México

salvador.cervantes@univa.mx

\section{Resumen}

El propósito de este artículo es realizar una revisión sistemática y un análisis bibliométrico de la producción científica relacionada con la Responsabilidad Social Universitaria, a través de una revisión en las bases de datos Web of Science y Scopus. Los registros obtenidos fueron analizados empleando la teoría de grafos y herramientas como bibliometrix, Sci Tool y Gephi; además, fueron presentados en tres categorías: hegemónicos, estructurales y recientes. Los resultados permitieron identificar tres perspectivas: medición, estratégico y conexión; también muestran que este campo de estudio es relativamente joven, en el cual el autor principal es Ricardo Gaete, mientras que François Vallaeys es el más citado. Adicionalmente, la región de mayor producción en el tema es Suramérica.

Palabras clave: Responsabilidad Social Universitaria, universidad, bibliometría, teoría de grafos.

\section{University Social Responsibility: a systematic review and a bibliometric analysis}

\section{Abstract}

The purpose of this article is to perform a systematic review and a bibliometric analysis of the scientific production related to University Social Responsibility, by means of a review in the Web of Science and Scopus databases. The records obtained were analyzed using the graph theory and tools such as bibliometrix, Sci ${ }^{2}$ Tool, and Gephi. In addition, they were presented in three categories: hegemonic, structural, and recent. The results allowed to identify three perspectives: measurement, strategic, and connection. They also show that this field of study is relatively young, in which the principal author is Ricardo Gaete, while François Vallaeys is the most cited. Additionally, the region with the highest production in the subject is South America.

Keywords: University Social Responsibility, university, bibliometrics, graph theory.

\section{Responsabilidade social universitária: revisão sistemática e análise bibliométrica}

\section{Resumo}

O objetivo deste artigo é realizar uma revisão sistemática e uma análise bibliométrica da produção científica relacionada à Responsabilidade Social Universitária, através de uma revisão nas bases de dados Web of Science e Scopus. Os registros obtidos foram analisados utilizando a teoria dos grafos e ferramentas como bibliometrix, Sci Tool e Gephi; Além disso, foram apresentados em três categorias: hegemônicos, estruturais e recentes. Os resultados permitiram identificar três perspectivas: mensuração, estratégica e conexão; Mostram também que esse campo de estudo é relativamente jovem, em que o autor principal é Ricardo Gaete, enquanto François Vallaeys é o mais citado. Além disso, a região com maior produção no assunto é a América do Sul.

Palavras-chave: Responsabilidade Social Universitária, universidade, bibliometria, teoria dos grafos.

*Autor para dirigir correspondencia. Dirigir a: Carrera $22 \mathrm{~N}^{\circ} 67 \mathrm{~A}-49$, Manizales, Colombia.

Clasificación JEL: M14.

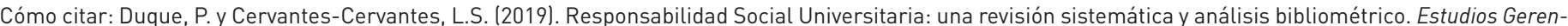
ciales, 35(153), 451-464. https://doi.org/10.18046/j.estger.2019.153.3389

DOI: https://doi.org/10.18046/j.estger.2019.153.3389

Recibido: 24-ene-2019

Aceptado: 4-dic-2019

Publicado: 20-dic-2019 


\section{Introducción}

El dinamismo de la investigación y producción científica en las últimas décadas ha despertado la necesidad de crear indicadores que permitan analizar el crecimiento y desarrollo de una disciplina o área del conocimiento (Bretón Lópéz y Buela Casal, 2003; Gómez y Solana, 2005). Este comportamiento ha dado lugar a los estudios bibliométricos que, apoyados en indicadores, buscan evaluar la ciencia y la productividad de los científicos (Moravcsik, 1989). Estos estudios adquieren valor dentro de la literatura ya que permiten dar una orientación a la comunidad académica respecto a los diferentes enfoques y avances de un área del conocimiento, además de diferentes elementos como la colaboración entre autores, la actividad científica por país o región, análisis de citación, el factor de impacto de las publicaciones y revistas (Sancho, 1990).

Por ejemplo, De Bakker, Groenewegen y Den Hond (2005) realizaron un análisis bibliométrico sobre Responsabilidad Social Corporativa y desempeño social, para un periodo de 30 años, encontrando que el progreso de la literatura en torno a este tema ha sido limitado por la carencia de un constructo generalizado de este concepto. Esto demuestra la relevancia y pertinencia de emplear técnicas de bibliometría en las investigaciones actuales.

La Responsabilidad Social Universitaria (en adelante RSU) es una política de gestión universitaria, que según Vallaeys (2014) se constituye como una evolución del concepto de Responsabilidad Social. Este campo de estudio ha despertado el interés de los investigadores en los últimos años; sin embargo, a pesar de que existen diferentes revisiones sobre el tema (De la Calle y Giménez, 2011; Larrán-Jorge y AndradesPeña, 2017; Mazzetti, 2014; Pacenza y Silva, 2013), no se ha realizado un estudio sistemático que muestre la evolución de este concepto, que permita identificar los documentos y autores principales, y que presente sus perspectivas.

En este sentido, De la Calle y Giménez (2011) efectuaron una aproximación al concepto, mediante la síntesis de la literatura asociada la RSU y la Responsabilidad Social del Universitario. Por un lado, Larrán-Jorge y Andrades-Peña (2017) revisaron las investigaciones asociadas al tema en 15 revistas académicas especializadas en educación superior. Por otro lado, Pacenza y Silva (2013) realizaron una revisión bibliométrica del tema; sin embargo, solo emplearon las bases de datos Scielo (Scientific Electronic Library Online) y Redalyc (Red de Revistas Científicas de América Latina y el Caribe). Por lo tanto, la presente investigación tiene como objetivo realizar una revisión sistemática basada en la teoría de grafos y efectuar un análisis bibliométrico de la producción científica relacionada con la RSU.

Para cumplir el objetivo en mención, inicialmente se realizó una búsqueda en las plataformas Web of Science len adelante WoS) y Scopus del tema University Social Responsibility. De estas bases de datos, se exportaron las referencias (200 en total) y posteriormente se realizó su procesamiento mediante la herramienta Bibliometrix, permitiendo hacer el análisis de índices como la cantidad de artículos, redes de cocitaciones y coautorías, productividad por países y por autores e impacto de las revistas. Posteriormente, se extraen todas las referencias de los artículos encontrados en las bases de datos (2.821 en total), esto se logra utilizando el aplicativo Sci2 Tool (Sci2 Team, 2009). Por último, se procede a realizar el análisis de la red generada a partir de todas las referencias, para ello se utiliza el aplicativo Gephi (Bastian, Heymann y Jacomy, 2009), logrando identificar los artículos más importantes de acuerdo a su impacto líndice de citación). Para la presentación de estos documentos se empleó un esquema de árbol; en la raíz se encuentran los documentos o artículos clásicos, en el tronco aquellos artículos que se consideran como estructurales, por último, en las hojas están los artículos más recientes; este esquema facilita comprender la evolución de la RSU, la importancia de los documentos y sus perspectivas.

El artículo se estructura en tres secciones adicionales a la introducción, en la primera se presenta la metodología empleada para el búsqueda, selección y procesamiento de los artículos base de la investigación, a partir del uso de herramientas bibliométricas. En la segunda, se expone el desarrollo de la investigación y los hallazgos. Por último, en la tercera parte del documento se socializan las principales conclusiones y hallazgos, al igual que se señalan las limitaciones del estudio y se efectúan recomendaciones para posteriores investigaciones de este tipo.

\section{Metodología}

Para la elaboración del presente artículo se realizó un estudio bibliométrico de la producción científica asociada al tema de RSU, la búsqueda de las investigaciones se efectuó en las bases de datos Web of Science (Clarivate) la cual cuenta con 18.000 revistas indexadas y Scopus (Elsevier) que actualmente agrupa 39.647 revistas indexadas, durante el periodo comprendido entre enero de 2001 y el 20 de junio de 2019. Estas dos bases de datos son consideradas las principales, ya que abarcan un amplio número de revistas a nivel mundial, además involucran investigaciones científicas en diversos campos del saber (Bar-Ilan, 2008, 2010; Gavel y Iselid, 2008; Vieira y Gomes, 2009).

La ecuación de búsqueda empleada fue la misma en ambas bases de datos: "University Social Responsibility", se realizó la consulta involucrando los temas, títulos, resúmenes y palabras claves, de esta manera se obtuvieron 116 artículos en Scopus y 84 en WoS (tabla 1).

Las referencias obtenidas por la búsqueda mencionada anteriormente fueron exportadas de WoS y Scopus, incluyendo contenido como: autor, título, año, DOI, fuente y referencias, que luego se integraron en una red. Este procedimiento incorpora artículos de diversas fuentes y revistas lindexadas

Tabla 1. Búsqueda y criterios

\begin{tabular}{lccc}
\hline Ecuación de búsqueda & Base de datos & Resultados & $\begin{array}{c}\text { Total de } \\
\text { artículos }\end{array}$ \\
\hline $\begin{array}{l}\text { University Social Respon- } \\
\text { sibility" }\end{array}$ & Scopus & 116 & \\
$\begin{array}{l}\text { Periodo de tiempo: Todos } \\
\text { los años }\end{array}$ & $\begin{array}{c}\text { Web of } \\
\text { Science }\end{array}$ & 84 & 200 \\
\hline
\end{tabular}

Fuente: elaboración propia 
y no indexadas), independientemente de la base de datos de donde se origina, al igual que su idioma, lo que ofrece una visión completa del campo de estudio y términos de producción académica más incluyente.

Para identificar los artículos más relevantes se empleó la metodología propuesta por Robledo, Osorio y López (2014) en la cual se ordenan los artículos en 3 categorías, la primera denominada la raíz, en la que se presentan los artículos con grado de entrada alto y salida cero; la segunda el tronco, allí se asocian los artículos con un grado de intermediación alto; la tercera y última las hojas, en la cual se localizan los artículos con alto grado de salida y un grado de entrada cero, también denominados las perspectivas (Robledo, Osorio y López, 2014]. Es importante mencionar que diversas investigaciones dan soporte a esta metodología, empleada en múltiples trabajos (Cervantes, Botero y Finkl, 2018; Díez, 2018; Hernández-Leal, Duque-Méndez y Moreno-Cadavid, 2017; Toro y del Rodríguez, 2017; Trujillo Salazar, 2017; Zuluaga et al., 2016).

Para la generación del mapa que muestra los artículos y la forma en que estos interactúan se utilizó la teoría de grafos propuesta por Leonhard Euler en 1941, la cual ha sido aplicada de manera amplia en investigaciones en las ciencias sociales (Wasserman y Faust, 1994). Esta técnica permite extraer información relevante de la topología y características de la red. Para ello se emplearon dos herramientas, la primera $\mathrm{Sci}^{2}$ Tool (Sci2 Team, 2009), en la cual se ingresa la lista de referencias y citas obtenidas de las bases de datos y se transforman en una red; la segunda herramienta es Gephi, la cual permite visualizar y analizar la red (Bastian et al., 2009). La metodología desarrollada se muestra en la figura 1.

Para el análisis bibliométrico se emplea la herramienta Bibliometrix, la cual es un código abierto que facilita el mapeo científico y se encuentra programado en el aplicativo "R" (Aria y Cuccurullo, 2017). Este código ha sido aplicado en diversas investigaciones y en diferentes disciplinas que requieren cartografía científica (Addor y Melsen, 2019; Almeida y de Paula, 2019; Alonso, Castiello y Mencar, 2018; Bond y Buntins, 2018).
Posterior a la generación de la red, se visualiza en la herramienta Gephi y se calculan los indicadores relacionados en la figura 1. El indicador Indegree hace referencia al número de veces que los artículos de la red han citado a uno en particular, el Outdegree hace referencia al número de veces que un artículo en particular cita a otros de la red, también se puede considerar como el número de enlaces que se desprenden de éste (Wallis, 2007). Dentro del aplicativo Gephi cada artículo está representado por un nodo y cada conexión por una arista.

Adicionalmente, se aplica el filtro de componente gigante, el cual busca aquellos nodos que están conectados entre sí de manera directa o indirecta, permitiendo eliminar nodos que no estén conectados con la comunidad principal (Bollobás, 1984). Finalmente, el último indicador en aplicarse es el Betweenness, éste permite calcular el número de rutas más cortas en el grafo que atraviesan un nodo (Freeman, 1977), el nodo resultante de este proceso contará con una centralidad e intermediación alta porque conecta varios nodos de la red (Ni, Sugimoto y Jiang, 2011), específicamente los artículos clásicos con los más recientes. En la figura 2 se visualizan la red inicial compuesta por 2821 nodos (referencias) y la red

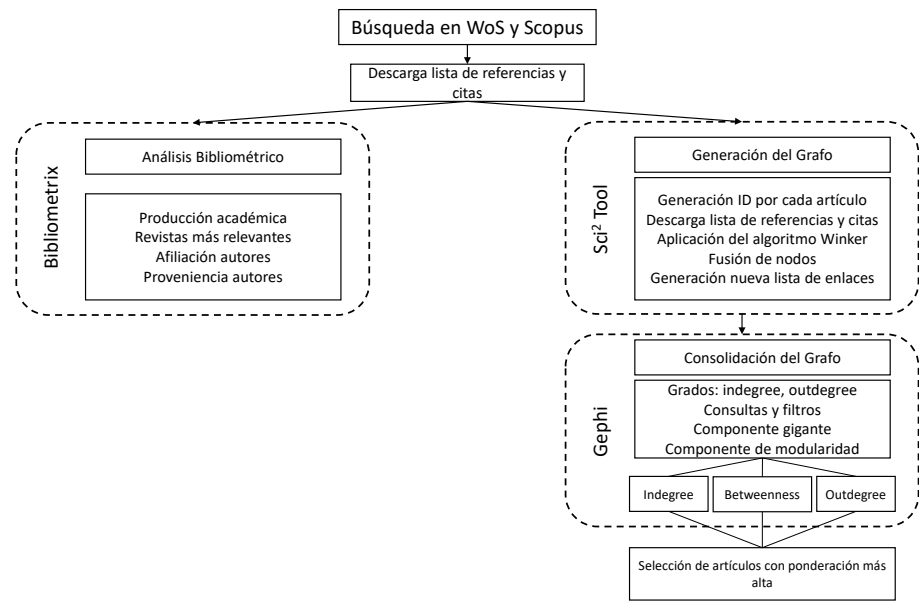

Figura 1. Procedimiento metodológico Fuente: elaboración propia.
Nodos: 2.821 Aristas: 3.142

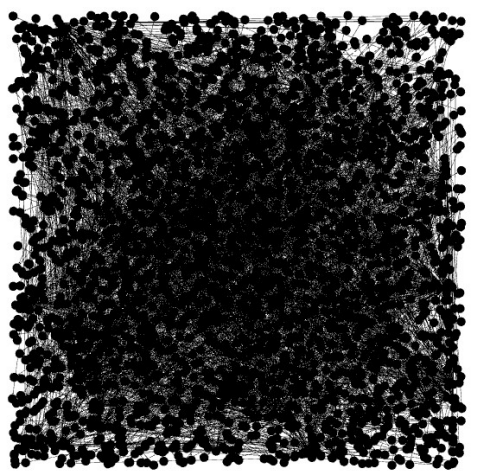

Nodos: 260 Aristas: 589

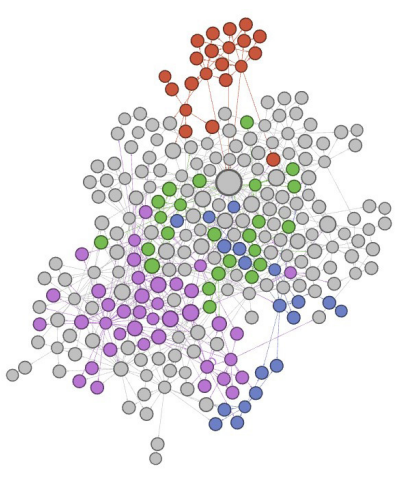

Figura 2. Extracción de la red

Fuente: elaboración propia. 
final compuesta por 260 nodos (referencias), donde la última se obtiene después de aplicar los filtros mencionados.

\section{Resultados}

En el presente apartado se relacionan elementos bibliométricos de la RSU como la producción científica, la discriminación por países, los autores más relevantes en términos de producción e índice de citaciones, así como las revistas más importantes en el campo. También se presenta la red y el árbol de la RSU, además, se plantea las perspectivas del área.

\subsection{Producción científica en RSU}

En la figura 3 se muestra la producción de artículos científicos publicados en las bases de datos WoS y Scopus asociados al tema de RSU entre los años 2001 y 2019, los cuales son 200 en total. Hasta el año 2014 se publicaron 56 documentos, siendo este periodo de tiempo el menos productivo; posterior a éste, la publicación de investigaciones enmarcadas en el tema ha aumentado de manera importante; durante el año 2018 (el periodo de más alta producción) se alcanzaron 49 artículos, lo que representa cerca del 25\% de las publicaciones totales. Como lo muestran las líneas de tendencia, se presenta un aumento del interés de la comunidad científica en esta área del conocimiento, lo que se refleja en una tasa anual de crecimiento del $32 \%$, es necesario aclarar que al momento de realizar el presente artículo habían transcurrido los primeros seis meses del año 2019, lo que explica que se contará con tan solo 18 publicaciones en ese periodo. También se debe resaltar que el comportamiento en el número de publicaciones en ambas bases de datos es muy similar en los últimos 5 años.

Al realizar el análisis de la producción científica por países, España se destaca en ambas bases de datos, con 34 publicaciones en Scopus y 29 en WoS; en la tabla 2 se puede observar el listado de los 10 países que lideran la producción académica mundial en RSU discriminados por base de datos; cabe resaltar que 6 países (España, Venezuela, Colombia, Chile, Brasil y Méxicol se encuentran en ambos listados.

Dentro de los 10 países de más alta producción el $80 \%$ son Latinoamericanos, lo que demuestra que esta región tiene un papel importante en cuanto a desarrollo investigativo en este campo. Colombia ocupa la segunda posición en el ámbito global con 29 publicaciones, seguido de Venezuela con 18, Chile y México con 14, Ecuador con 11, Perú con 9, Romania con 8, Brasil con 7, y por último Estados Unidos con 7 (figura 4).

En relación con los autores, la tabla 3 proporciona el listado de los autores más relevantes, clasificándolos por el número de documentos publicados en cada base de datos, además, se relaciona su índice $\mathrm{h}(H$-index), este indicador es empleado para caracterizar la producción científica de cada

Tabla 2. Producción de artículo en RSU por países

\begin{tabular}{lclc}
\hline & \multicolumn{2}{c}{ Base de datos } \\
\hline País & $\begin{array}{c}\text { Número de } \\
\text { documentos }\end{array}$ & País & $\begin{array}{c}\text { Número de } \\
\text { documentos }\end{array}$ \\
\hline España & 34 & España & 29 \\
Venezuela & 14 & Colombia & 17 \\
Colombia & 12 & México & 10 \\
Chile & 9 & Ecuador & 9 \\
Romania & 6 & Perú & 6 \\
Brasil & 5 & Chile & 5 \\
Estados Unidos & 5 & Venezuela & 4 \\
México & 4 & Brasil & 2 \\
Rusia & 4 & Malasia & 2 \\
Australia & 3 & Polonia & 2 \\
\hline Fuente: & & &
\end{tabular}

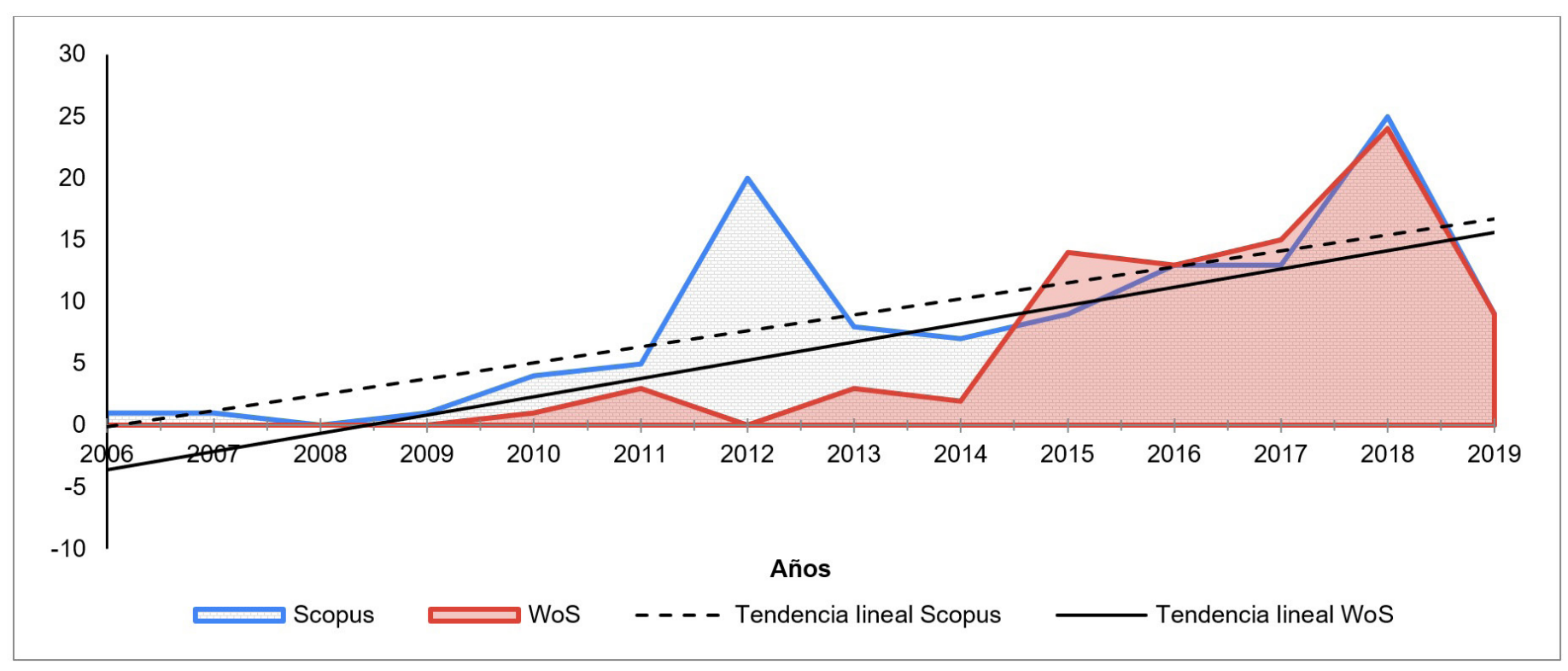

Figura 3. Producción científica anual en RSU Fuente: elaboración propia. 


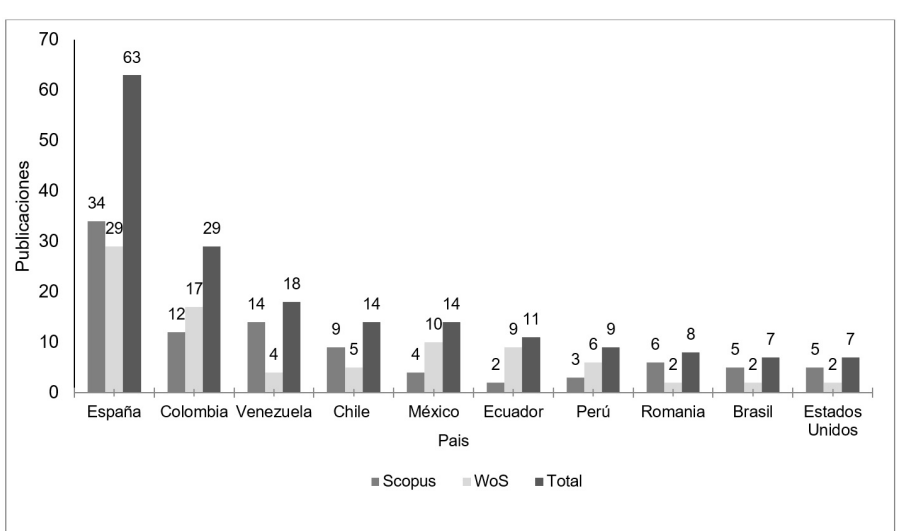

Figura 4. 10 Países con mayor producción en RSU

Fuente: elaboración propia.

Tabla 3. Autores más relevantes en la producción de artículos sobre RSU

\begin{tabular}{|c|c|c|c|c|c|}
\hline \multicolumn{2}{|c|}{ Scopus } & \multirow[b]{2}{*}{$\begin{array}{c}\text { Índice } \\
\mathrm{h}\end{array}$} & \multicolumn{2}{|l|}{ WoS } & \multirow[b]{2}{*}{$\begin{array}{l}\text { Índice } \\
\text { h }\end{array}$} \\
\hline Autor & $\begin{array}{l}\text { Número } \\
\text { de publi- } \\
\text { caciones }\end{array}$ & & Autor & $\begin{array}{l}\text { Número } \\
\text { de publi- } \\
\text { caciones }\end{array}$ & \\
\hline Quezada, R.G. & 5 & 3 & $\begin{array}{l}\text { Quezada, } \\
\text { R.G. }\end{array}$ & 4 & 1 \\
\hline Chang, E. & 3 & 31 & $\begin{array}{l}\text { Hernández, } \\
\text { B. }\end{array}$ & 3 & 0 \\
\hline Esfijani, A. & 3 & 4 & Vallaeys, F. & 3 & 1 \\
\hline $\begin{array}{l}\text { Abdel-Hameid, } \\
\text { S.O. }\end{array}$ & 2 & 0 & $\begin{array}{l}\text { Rodríguez, } \\
\text { N.A. }\end{array}$ & 2 & 0 \\
\hline Aza, C.L. & 2 & 2 & $\begin{array}{l}\text { López- } \\
\text { Noriega, } \\
\text { M.D. }\end{array}$ & 2 & 0 \\
\hline Badri, A.B. & 2 & 2 & $\begin{array}{l}\text { Moreno, } \\
\text { J.R.V }\end{array}$ & 2 & 0 \\
\hline Bocean, C. & 2 & 1 & $\begin{array}{l}\text { Martí No- } \\
\text { guera, J.J. }\end{array}$ & 2 & 1 \\
\hline $\begin{array}{l}\text { Gallardo- } \\
\text { Vázquez, D. }\end{array}$ & 2 & 6 & Rajab, A. & 2 & 0 \\
\hline Hussain, F.K. & 2 & 23 & Shaari, R. & 2 & 0 \\
\hline Lanero, A. & 2 & 5 & $\begin{array}{l}\text { Zalthen- } \\
\text { Hernandez, } \\
\text { L. }\end{array}$ & 2 & 0 \\
\hline
\end{tabular}

Fuente: elaboración propia

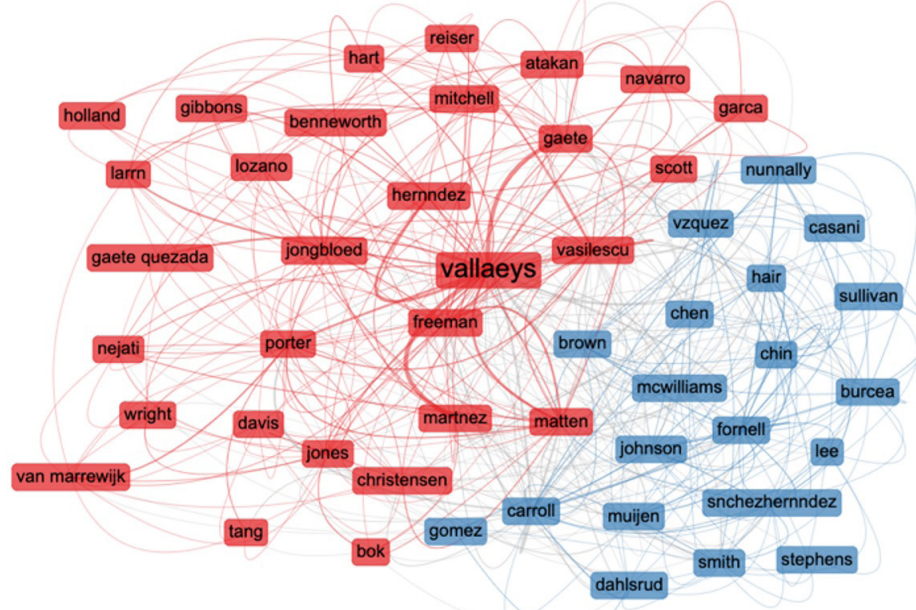

Figura 5. Red cocitaciones

Fuente: elaboración propia. investigador (Hirsch, 2005). Ricardo Gaete Quezada lidera la producción tanto en Scopus como en WoS, con un total de 9 artículos publicados en ambas bases de datos, sin embargo, el $\mathrm{H}$-index de este autor no es el mejor dentro de este listado; particularmente, Elizateth Chang y Farookh Hussain, quienes son coautores, cuentan con un indicador mucho más alto (tabla 3).

Los documentos con mayor índice de impacto en Scopus y WoS son: "Developing university social responsibility: A model for the challenges of the new civil society" (Vasilescu, Barna, Epure y Baicu, 2010) citado 34 veces en Scopus y 138 en Google Scholar, y "Online disclosure of university social responsibility: a comparative study of public and private US universities" (Garde Sánchez, Rodríguez Bolívar y López-Hernández, 2013) citado 23 veces en Scopus y 42 en Google Scholar.

Las publicaciones en la base de datos Scopus se concentran en las áreas de ciencias sociales $(34,5 \%)$, negocios, gestión y contabilidad $(17,5 \%)$, economía, econometría y finanzas $(12,1 \%)$ y artes y humanidades $(10,7 \%)$. En WoS se centralizan en categorías como investigación en educación $(38 \%)$ y ciencias sociales $(21,5 \%)$, lo que muestra que la clasificación empleada por la WoS es más homogénea que la empleada por Scopus, además, ofrece un campo mucho más específico para las investigaciones enmarcadas en RSU (investigación educativa).

Respecto a la red de cocitaciones (figura 5), se observa que el autor principal es François Vallaeys, quien es el más citado dentro de la red y con el mayor número de conexiones. Además, se pueden evidenciar dos clústeres de autores, el primero y más grande integrado por algunos investigados como: Vallaeys, Gaete-Quezada, Vasilescu, Martínez, Christensen, Hernández, Matten, Benneworth y Mitchell; el segundo compuesto entre otros por: Brown, Gómez, McWilliams, Hair, Vázquez, Johnson y Fornell.

En la tabla 4 se relacionan las diez revistas científicas en las cuales se han publicado más artículos asociados al tema de RSU dentro de las bases de datos Scopus y WoS, además, se presenta el índice h y el cuartil en el que se encuentra cada una de éstas. Tan solo una de las revistas se encuentra en Q1, otra en Q2, cinco en $\mathrm{Q} 3$ y tres no registran cuartil.

\subsection{Análisis de la red}

Los resultados obtenidos de la ecuación de búsqueda produjeron una red de 2.821 nodos y 3.142 enlaces; posterior al procesamiento de esta red inicial se obtuvo una red compuesta de 260 nodos y 589 enlaces (figura 2). Para seleccionar los artículos objeto de análisis se empleó el criterio de puntuación más alta en los indicadores Indegree Igrado de entrada), Betweenness (intermediación) y Outdegree (grado de salidal. A partir de lo anterior, se eligieron 40 artículos en total para esta revisión: los 10 artículos con el más alto grado de entrada, 15 con el mayor grado de intermediación y 15 con el más alto grado de salida; estos artículos representan información relevante en un amplio periodo de tiempo. Los documentos clásicos o hegemónicos son aquellos que tienen un valor alto de Indegree y cero de Outdegree, los actuales son los que presentan un valor alto de Outdegree y cero de 
Indegree, y los estructurales son los que tienen un alto valor en Betweenness, es decir, lo que conectan los documentos clásicos con los actuales. Los documentos seleccionados se presentan dentro de la estructura de un árbol, en la cual los documentos que son considerados clásicos se encuentran en las raíces, los estructurales el tronco y los actuales en las hojas (figura 6).

La figura 7 muestra la red de la RSU (compuesta por 260 documentos) a la cual se aplicó el algoritmo de modularidad

Tabla 4. Revistas científicas más importantes

\begin{tabular}{|c|c|c|c|c|}
\hline Revista & Artículos & $\begin{array}{l}\text { Índice } \\
\mathrm{h}\end{array}$ & Cuartil & $\begin{array}{c}\text { Base de } \\
\text { datos }\end{array}$ \\
\hline Opción & 12 & 11 & Q3 & Scopus \\
\hline $\begin{array}{l}\text { World review of entrepre- } \\
\text { neurship management and } \\
\text { sustainable development }\end{array}$ & 9 & 13 & Q3 & Scopus \\
\hline $\begin{array}{l}\text { Revista digital de investiga- } \\
\text { ción en docencia universita- } \\
\text { ria RIDU }\end{array}$ & 9 & 1 & - & WoS \\
\hline $\begin{array}{l}\text { Revista venezolana de } \\
\text { gerencia }\end{array}$ & 4 & 7 & Q3 & Scopus \\
\hline Espacios & 3 & 12 & Q3 & Scopus \\
\hline $\begin{array}{l}\text { International review on pu- } \\
\text { blic and nonprofit marketing }\end{array}$ & 3 & 11 & Q3 & Scopus \\
\hline $\begin{array}{l}\text { EUREKA Revista científica de } \\
\text { psicología }\end{array}$ & 3 & 0 & - & WoS \\
\hline Revista CONRADO & 3 & 1 & - & WoS \\
\hline $\begin{array}{l}\text { Education policy analysis } \\
\text { archives }\end{array}$ & 2 & 40 & Q1 & Scopus \\
\hline $\begin{array}{l}\text { International Journal of } \\
\text { Management }\end{array}$ & 2 & 44 & Q2 & Scopus \\
\hline
\end{tabular}

de clase, el cual permite visualizar dentro de la red diferentes comunidades (clúster) integradas por nodos densamente conectados (Blondel, Guillaume, Lambiotte y Lefebvre, 2008), en este caso se identificaron tres perspectivas. Los nodos de mayor tamaño son los documentos que se han citado con mayor frecuencia dentro de la red, esto implica que son considerados los más relevantes en este campo del conocimiento. En este caso se señalaron los 4 más importantes.

\subsection{Documentos clásicos}

Los documentos que son considerados hegemónicos (tabla 5) pueden clasificarse en dos grupos, el primero en el cual se encuentran autores que han realizado aportes considerables a la Responsabilidad Social en términos generales (Bowen, 1960; Carroll, 1999, 1979; Freeman y Mcvea, 1984; Portery Kramer, 2006), el segundo integrado por investigaciones sobre Responsabilidad Social enmarcadas en el sector universitario; es allí donde se encuentran cinco trabajos elaborados por François Vallaeys, lo que corrobora los resultados presentando en la red de cocitaciones y ratifica el protagonismo de este autor en el campo de estudio.

El documento de mayor impacto en el tema de RSU es “Responsabilidad Social Universitaria: manual de primeros pasos", este libro publicado por Vallaeys (2009) presenta una herramienta de autodiagnóstico compuesta por cuatro ejes (campus responsable, gestión social del conocimiento, participación social y formación profesional y ciudadanal que permiten integrar diversas áreas como las funciones y los diferentes grupos de interés de estas instituciones; este instrumento también facilita el desarrollo estrategias de RSU

Fuente: elaboración propia

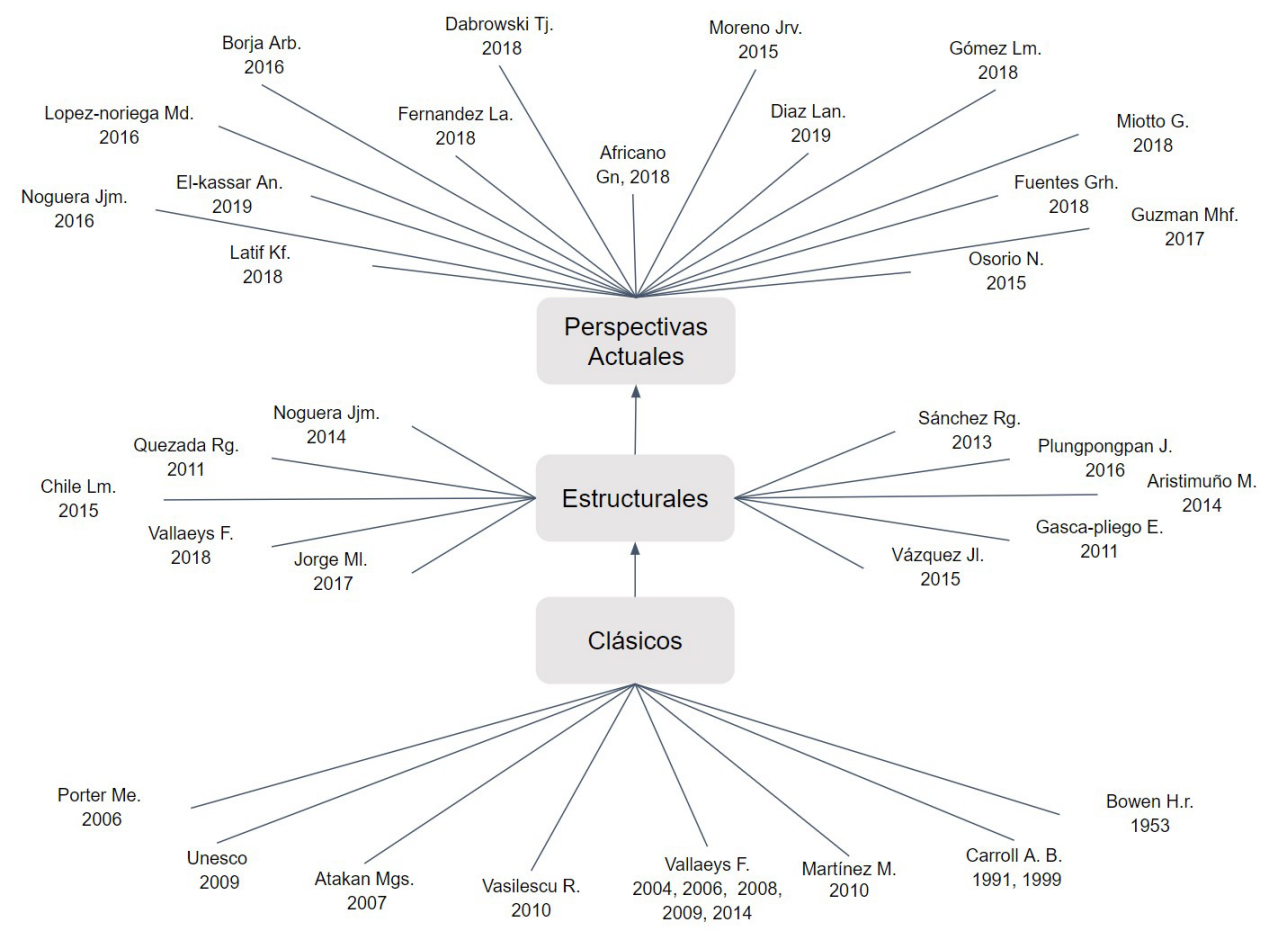

Figura 6. Árbol de la RSU

Fuente: elaboración propia. 


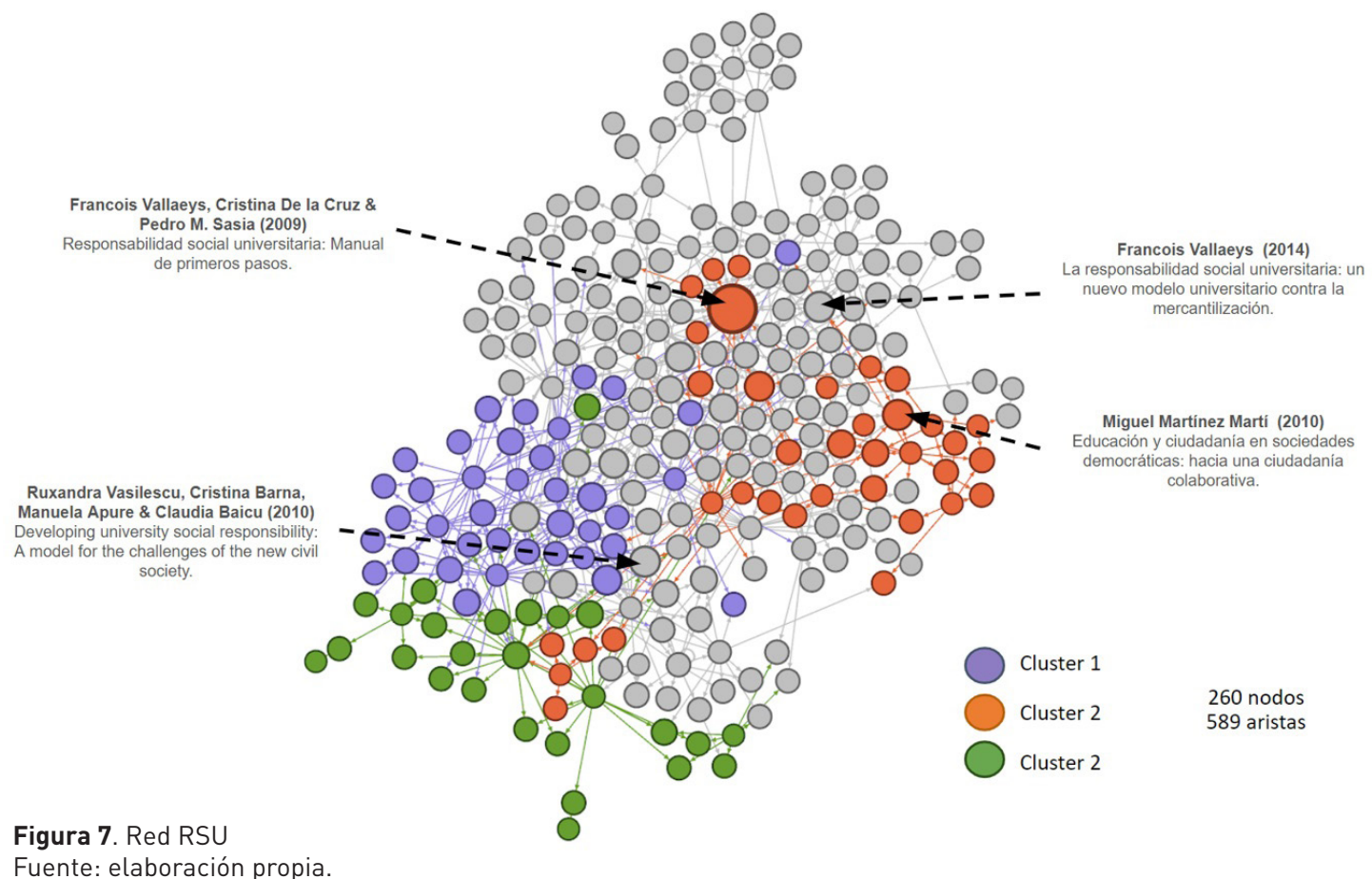

Tabla 5. Documentos hegemónicos

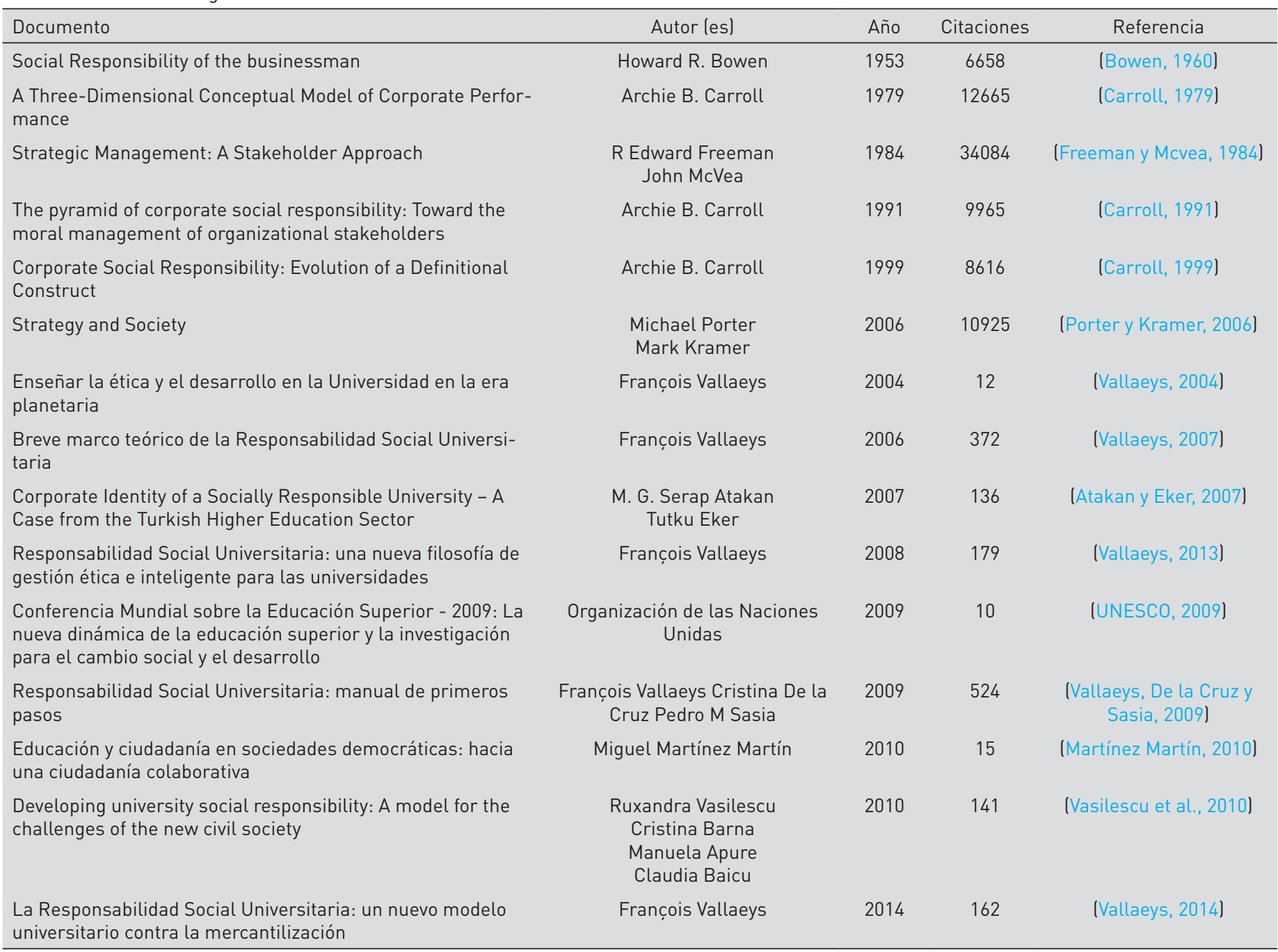

Fuente: elaboración propia 
que generan mayores impactos organizacionales, educativos, cognitivos y sociales.

Vallaeys realiza otros aportes considerables al campo de estudio:

- En el año 2006 publica el artículo titulado “Breve marco teórico de la Responsabilidad Social Universitaria” en el cual plantea una reflexión sobre lo que significa el concepto de RSU y sus implicaciones, además, explica su evolución, al pasar de ser sencillas iniciativas de proyección social, hasta lo que hoy representa; también expone los desafíos que deben enfrentar todas las iniciativas que se emprendan en este tema.

- En el año 2008 publica el artículo titulado “Responsabilidad Social Universitaria: una nueva filosofía de gestión ética e inteligente para las universidades", en este documento el autor expone que la RSU debe dejar de verse como un agregado o un conjunto de acciones encaminadas a la proyección social, y la presenta como un modelo de gestión, que puede generar a las instituciones innovación académica, coherencia institucional y pertinencia social.

- Finalmente, en el año 2014 publica el artículo “La Responsabilidad Social Universitaria: un nuevo modelo universitario contra la mercantilización", en este analiza el modelo de gestión de las instituciones de educación superior basado en la RSU y lo presenta como una herramienta para reivindicar los valores y verdadera finalidad de las universidades. Según el autor, el desprestigio que enfrentan estas organizaciones se debe a la visión de "universidad empresarial" lo que las convierte en organizaciones con fines

Tabla 6. Documentos estructurales

\begin{tabular}{|c|c|c|c|}
\hline Documento & Descripción & Metodología & Referencia \\
\hline $\begin{array}{l}\text { Construir ciudadanía desde } \\
\text { las universidades, Responsa- } \\
\text { bilidad Social Universitaria y } \\
\text { desafíos ante el siglo XXI }\end{array}$ & $\begin{array}{l}\text { Es un documento reflexivo que aborda el rol que deben desempeñar las } \\
\text { universidades como agentes de transformación y ratificación del modelo } \\
\text { democrático, mediante la formación de ciudadanos completos y participativos, } \\
\text { preocupados por las necesidades sociales. }\end{array}$ & Teórico & $\begin{array}{l}\text { (Gasca-Pliego y } \\
\text { Olvera-García, } \\
\text { 2011) }\end{array}$ \\
\hline $\begin{array}{l}\text { Responsabilidad Social Uni- } \\
\text { versitaria. Su gestión desde } \\
\text { la perspectiva de directivos y } \\
\text { docentes. Estudio de caso: una } \\
\text { pequeña universidad latinoa- } \\
\text { mericana }\end{array}$ & $\begin{array}{l}\text { A través de un caso de estudio de la Universidad Nacional Experimental de } \\
\text { Guayana en Venezuela se examina la percepción de los directivos y docentes } \\
\text { de esta institución respecto a la RSU, mediante la medición de cinco facto- } \\
\text { res (cognitivo-ambiental, currículo y clima organizacional, comportamiento } \\
\text { organizacional, selección de stakeholders y planificación hacia el compromiso } \\
\text { social) }\end{array}$ & Empírico & $\begin{array}{l}\text { (Aristimuño } \\
\text { y Rodriguez } \\
\text { Monroy, 2014] }\end{array}$ \\
\hline $\begin{array}{l}\text { Students' experiences of } \\
\text { university social responsibility } \\
\text { and perceptions of satisfaction } \\
\text { and quality of service }\end{array}$ & $\begin{array}{l}\text { Mediante un instrumento aplicado a los estudiantes de la Universidad de León } \\
\text { en España se identificaron los factores que definen la percepción que estos } \\
\text { tienen sobre la RSU. }\end{array}$ & Empírico & $\begin{array}{l}\text { (Vázquez, Lane- } \\
\text { ro y Aza, 2015) }\end{array}$ \\
\hline $\begin{array}{l}\text { Online disclosure of university } \\
\text { social responsibility: a com- } \\
\text { parative study of public and } \\
\text { private US universities }\end{array}$ & $\begin{array}{l}\text { Es un estudio comparativo entre universidades públicas y privadas en Estados } \\
\text { Unidos, en el cual se analiza si estas instituciones integran dentro de sus } \\
\text { actividades prácticas de RSU, además, si utilizan la RS como un factor diferen- } \\
\text { ciador. }\end{array}$ & Empírico & $\begin{array}{c}\text { (Garde Sánchez } \\
\text { et al., 2013) }\end{array}$ \\
\hline $\begin{array}{l}\text { Analysing the literature on } \\
\text { university social responsibility: } \\
\text { A review of selected higher } \\
\text { education journals }\end{array}$ & Es una revisión de la literatura sobre RSU durante el periodo 2000 a 2015. & Empírico & $\begin{array}{l}\text { (Larrán-Jorge } \\
\text { y Andrades-Pe- } \\
\text { ña, 2017) }\end{array}$ \\
\hline $\begin{array}{l}\text { La Responsabilidad Social } \\
\text { Universitaria como desafío } \\
\text { para la gestión estratégica de } \\
\text { la Educación Superior: } \\
\text { el caso de España }\end{array}$ & $\begin{array}{l}\text { Es un análisis del concepto de RSU y una clasificación del mismo a través de } \\
\text { tres perspectivas: gerenciales, transformadoras y normativas. Se analiza el } \\
\text { desarrollo de este concepto en España }\end{array}$ & Empírico & $\begin{array}{l}\text { (Gaete Queza- } \\
\text { da, 2011) }\end{array}$ \\
\hline $\begin{array}{l}\text { Responsabilidad Social } \\
\text { Universitaria: influencia de } \\
\text { valores y empatía en la autoa- } \\
\text { tribución de comportamientos } \\
\text { socialmente responsables }\end{array}$ & $\begin{array}{l}\text { Es una revisión de la RSU desde la psicología, analiza cómo la formación per- } \\
\text { mite adquirir a los estudiantes comportamientos socialmente responsables. } \\
\text { La investigación evaluó tres escalas: valores humanos, empatía multidimen- } \\
\text { sional y autoatribución de comportamientos socialmente responsables. }\end{array}$ & Empírico & $\begin{array}{l}\text { (Martí Noguera, } \\
\text { Martí-Vilar y } \\
\text { Almerich, 2014] }\end{array}$ \\
\hline
\end{tabular}


de lucro, y cualquier fin diferente a la universalidad termina destruyendo a estas instituciones.

Otras publicaciones que se consideran clásicas dentro de este campo de estudio son: “Developing university social responsibility: A model for the challenges of the new civil society" y "Corporate Identity of a Socially Responsible University - A Case from the Turkish Higher Education Sector", las cuales a diferencia de las investigaciones de Vallaeys son desarrolladas fuera de Latinoamérica. En el caso de la primera se exhibe un modelo general de RSU en el contexto de la globalización, considerando las realidades y los desafíos del sistema de educación superior en Rumania; la segunda es un estudio que describe las iniciativas de Responsabilidad Social de una institución de educación superior en Turquía.

Martínez Martí (2010) realiza una propuesta pedagógica sobre formación de ciudadanos en sociedades democráticas, plurales y en época de globalización, argumentando que la ciudadanía activa y colaborativa se logra desde la educación formal, no formal e incluso la informal.

Finalmente, la Organización para las Naciones Unidas para la Educación, la Ciencia y la Cultura (UNESCO) en la conferencia celebrada en París en el año 2009, analiza el papel de la educación superior y la investigación en el cambio social y desarrollo, y emite un comunicado que plantea las responsabilidades de la educación superior, sus retos y las dinámicas que debe enfrentar para contribuir al cumplimiento de los Objetivos de Desarrollo del Milenio.

\subsection{Documentos estructurales}

Los documentos con mayor interés son los que vinculan los recientes con los clásicos, son los que presentan mayor tasa de centralidad, es decir, son citados de manera importante y al mismo tiempo citan los documentos hegemónicos, estos artículos conectan la red.

Dentro de los documentos que hacen parte del tronco (estructurales) del árbol de la RSU se identificó una tendencia a desarrollar investigaciones de corte empírico, algunos se interesaron en construir y aplicar instrumentos que permitieran evaluar cómo los diferentes grupos de interés percibían las prácticas de responsabilidad social desarrolladas por las universidades (Aristimuño y Rodriguez Monroy, 2014; Larrán Jorge y Andrades Peña, 2017; Plungpongpan, Tiangsoongnern y Speece, 2016; Vázquez, Lanero y Aza, 2015). También se identificaron documentos completamente teóricos como el publicado por Gasca-Pliego y Olvera-García (2011), en el cual de manera reflexiva se analiza el papel que desempeñan las universidades como agentes del cambio social. En la tabla 6 se presentan los documentos y la descripción de cada uno.

\subsection{Documentos recientes}

Los documentos con mayor grado de salida son los que emplean como referentes a los que hacen parte de las raíces y el tronco, en este caso reciben el nombre de hojas. En esta sección hay una tendencia a desarrollar investigaciones empíricas que pretenden determinar cómo son percibidas las prácticas de Responsabilidad Social de las universidades por parte de los diferentes grupos de interés, además, de proponer y validar escalas para medirlas. En la tabla 7 se exponen los documentos que integran esta categoría y se presentan elementos como el objetivo, la metodología y la revista en la cual fueron publicados.

Tabla 7. Documentos recientes

\begin{tabular}{|c|c|c|c|c|}
\hline Documento & Objetivo & Metodología & $\begin{array}{l}\text { Revista / } \\
\text { Editor }\end{array}$ & Referencia \\
\hline $\begin{array}{l}\text { The Development and Vali- } \\
\text { dation of Stakeholder-Ba- } \\
\text { sed Scale for Measuring } \\
\text { University Social Respon- } \\
\text { sibility (USR) }\end{array}$ & Desarrollar y validar una escala de medición de la RSU & Empírico & $\begin{array}{l}\text { Social Indica- } \\
\text { tors Research } \\
\text { (Q1) }\end{array}$ & (Latif, 2017) \\
\hline $\begin{array}{l}\text { El desarrollo regional } \\
\text { como responsabilidad so- } \\
\text { cial de la universidad. Caso } \\
\text { ecoturismo en la provincia } \\
\text { de Tungurahua }\end{array}$ & $\begin{array}{l}\text { Analizar el rol de la universidad en el desarrollo regional mediante un } \\
\text { caso de estudio }\end{array}$ & Empírico & $\begin{array}{c}\text { Revista } \\
\text { colombiana } \\
\text { de ciencias } \\
\text { sociales }\end{array}$ & $\begin{array}{l}\text { (Martí Nogue- } \\
\quad \text { ra, 2016) }\end{array}$ \\
\hline $\begin{array}{l}\text { Student-university identi- } \\
\text { fication and loyalty through } \\
\text { social responsibility: A } \\
\text { cross-cultural analysis }\end{array}$ & $\begin{array}{l}\text { Examinar el efecto de la RSU sobre la lealtad de los estudiantes y el } \\
\text { efecto moderador de la importancia percibida de la RSU }\end{array}$ & Empírico & $\begin{array}{l}\text { International } \\
\text { Journal of } \\
\text { Educational } \\
\text { Management } \\
\text { [Q2] }\end{array}$ & $\begin{array}{l}\text { (Vázquez et } \\
\text { al., 2015) }\end{array}$ \\
\hline $\begin{array}{l}\text { Responsabilidad Social } \\
\text { Universitaria. Tratamiento } \\
\text { en la UMET }\end{array}$ & $\begin{array}{l}\text { Analizar los elementos que intervienen en la puesta en práctica de la } \\
\text { RSU en la Universidad Metropolitana del Ecuador }\end{array}$ & Empírico & Conrado & $\begin{array}{l}\text { (Brivio Borja } \\
\text { y Montano } \\
\text { Rodríguez, } \\
\text { 2016) }\end{array}$ \\
\hline
\end{tabular}


Tabla 7. Documentos recientes (continuación)

\begin{tabular}{|c|c|c|c|c|}
\hline $\begin{array}{l}\text { Discursos representacio- } \\
\text { nales sobre transferencia } \\
\text { social del conocimiento } \\
\text { en la Universidad de } \\
\text { Carabobo }\end{array}$ & $\begin{array}{l}\text { Generar una interpretación sobre las representaciones sociales de } \\
\text { docentes de la Universidad de Carabobo (Venezuela) asociado a la } \\
\text { transferencia social de conocimiento }\end{array}$ & Teórico & Orbis & $\begin{array}{l}\text { IOsorio y } \\
\text { Zambrano, } \\
\text { 2015) }\end{array}$ \\
\hline $\begin{array}{l}\text { La comunicación y medi- } \\
\text { ción de la Responsabilidad } \\
\text { Social Universitaria: Redes } \\
\text { sociales y propuesta de } \\
\text { indicadores }\end{array}$ & $\begin{array}{l}\text { Analizar la comunicación de las prácticas de Responsabilidad Social } \\
\text { de las universidades Españolas a través de Twitter }\end{array}$ & Empírico & $\begin{array}{l}\text { Revista } \\
\text { Digital de } \\
\text { Investigación } \\
\text { en Docencia } \\
\text { Universitaria }\end{array}$ & $\begin{array}{l}\text { (Aldeanueva } \\
\text { y Arrabal, } \\
\text { 2018) }\end{array}$ \\
\hline $\begin{array}{l}\text { Practicando la Responsa- } \\
\text { bilidad Social Universitaria } \\
\text { en el Caribe: Perspectivas } \\
\text { de los públicos internos }\end{array}$ & $\begin{array}{l}\text { Analizar las perspectivas de públicos internos sobre las iniciativas } \\
\text { y procesos de responsabilidad social en una universidad privada en } \\
\text { Puerto Rico }\end{array}$ & Empírico & $\begin{array}{c}\text { Revista } \\
\text { Digital de } \\
\text { Investigación } \\
\text { en Docencia } \\
\text { Universitaria }\end{array}$ & $\begin{array}{l}\text { (Gómez, } \\
\text { Alvarado Na- } \\
\text { veira y Pujol } \\
\text { Bernabel, } \\
\text { 2018] }\end{array}$ \\
\hline $\begin{array}{l}\text { University Social Respon- } \\
\text { sibility Strategies }\end{array}$ & $\begin{array}{l}\text { Investigar cómo las instituciones de educación superior desarrollan } \\
\text { sus estrategias de responsabilidad social }\end{array}$ & Empírico & e-mentor & $\begin{array}{l}\text { (Dsntowski, } \\
\text { Brdulak, } \\
\text { Jastrzebska y } \\
\text { Legutko-Ko- } \\
\text { bus, 2018) }\end{array}$ \\
\hline $\begin{array}{l}\text { La transferencia tecno- } \\
\text { lógica como práctica de } \\
\text { Responsabilidad Social } \\
\text { Universitaria }\end{array}$ & $\begin{array}{l}\text { Definir a partir de una revisión bibliográfica cómo se puede entender } \\
\text { la transferencia tecnológica como práctica de RSU }\end{array}$ & Teórico & Arbor (Q2) & $\begin{array}{l}\text { (Naranjo Afri- } \\
\text { cano y Mejia } \\
\text { Reatiga, } \\
\text { 2018) }\end{array}$ \\
\hline $\begin{array}{l}\text { Hacia una definición lati- } \\
\text { noamericana de Respon- } \\
\text { sabilidad Social Universi- } \\
\text { taria. Aproximación a las } \\
\text { preferencias conceptuales } \\
\text { de los universitarios }\end{array}$ & $\begin{array}{l}\text { Identificar cuánto han avanzado los universitarios latinoamericanos } \\
\text { en la comprensión del paradigma de la RSU }\end{array}$ & Empírico & $\begin{array}{l}\text { Educación } \\
\text { XX1 (Q2) }\end{array}$ & $\begin{array}{l}\text { (Vallaeys } \\
\text { y Álvarez } \\
\text { Rodríguez, } \\
\text { 2019) }\end{array}$ \\
\hline $\begin{array}{l}\text { La inclusión de grupos } \\
\text { de atención prioritaria en } \\
\text { proyectos de vinculación } \\
\text { de la sociedad }\end{array}$ & $\begin{array}{l}\text { Analizar las bases teóricas y prácticas sobre la inclusión de los } \\
\text { grupos prioritarios para que se integren a la sociedad, mediante los } \\
\text { diferentes proyectos de vinculación que ofrece la Universidad Metro- } \\
\text { politana del Ecuador (UMET)" }\end{array}$ & Empírico & Conrado & $\begin{array}{l}\text { (Novillo Díaz, } \\
\text { 2019) }\end{array}$ \\
\hline $\begin{array}{l}\text { Responsabilidad Social } \\
\text { Universitaria "Una aproxi- } \\
\text { mación desde la percep- } \\
\text { ción de la colectividad } \\
\text { académica" }\end{array}$ & $\begin{array}{l}\text { Identificar el significado de Responsabilidad Social Universitaria } \\
\text { (RSU) percibido por parte de sus grupos de interés, especialmente, } \\
\text { de los clientes internos. }\end{array}$ & Empírico & Hermes & $\begin{array}{c}\text { (Flórez } \\
\text { Guzmán, } \\
\text { Rubio-Rodri- } \\
\text { guez, Rodrad } \\
\text { Cooperativ y } \\
\text { UniveCurvelo } \\
\text { Hasssl, 2017] }\end{array}$ \\
\hline
\end{tabular}

Fuente: elaboración propia

Martí Noguera (2016) sugiere que el deber y responsabilidad de la universidad es generar e incentivar investigaciones aplicadas que puedan ser transferidas en términos sociales, además, considera que la RSU no se limita a investigar, publicar y formar, sino a buscar formas de comprender, adaptar y transferir el conocimiento. Vallaeys y Álvarez (2019) sugieren que el concepto de RSU, visto de desde la gestión de impactos, toma cada vez más fuerza. En su investigación titulada "Hacia una definición latinoamericana de Responsabilidad Social Universitaria. Aproximación a las preferencias conceptuales de los universitarios" concluyen que en el contexto latinoamericano se está superando la visión reductora de compromiso social universitario asumido solamente desde la extensión voluntaria. Henríque, Vallaeys y Castrillón (2018) analizan cómo debe organizarse la universidad para ser socialmente responsable y cumplir su proyecto institucional, como resultado proponen un modelo teórico de aprendizaje universitario para la responsabilidad social.

A partir de lo anterior, se identificaron tres perspectivas dentro de los documentos recientes que se analizarán a continuación (figura 8): 
- Perspectiva 1: medición de la RSU

Esta perspectiva representa el $7 \%$ del total de artículos de la red y está integrada por investigaciones que centran su atención en la comunicación y medición de la RSU.

Algunos de los resultados de las investigaciones presentes en esta perspectiva se presentan a continuación: Aldeanueva y Arrabal (2018) analizaron la comunicación de las prácticas de Responsabilidad Social de las universidades españolas a través de Twitter, para ello emplearon 50 variables asociadas con el tema la RSU, concluyendo que los conceptos semánticos asociados a la Responsabilidad Social Corporativa no forman parte de las estrategias de comunicación exterior de las universidades. Garde Sánchez et al. (2013) efectúan un estudio comparativo entre universidades públicas y privadas en Estados Unidos, en el cual se analiza si estas instituciones integran dentro de sus actividades prácticas de RSU, además, si las utilizan como un factor diferenciador. Latif (2017) diseñó y validó una escala de medición para la RSU, compuesta por 7 dimensiones. Gómez, Alvarado y Pujol (2018) realizan su aporte a la literatura sobre el tema a través de la aplicación de un instrumento ajustado al propuesto por Vallaeys et al. (2009) específicamente desde la perspectiva de los públicos internos (docentes, administrativos y estudiantes).

\section{- Perspectiva 2: estratégico}

Esta perspectiva representa el 5\% del total de artículos de la red y la caracterizan investigaciones que dan un enfoque estratégico a la RSU, dimensionándola como un factor que permite diferenciar una institución de la otra.

Algunos de los resultados de las investigaciones presentes en esta perspectiva señalan: Gaete Quezada (2011) afirma que la RSU se constituye como un desafío estratégico para las universidades, sin embargo, aún no existe un consenso de su significado ni aplicación, para ello propone tres perspectivas: gerenciales, transformadoras y normativas, que facilitan la identificación de las iniciativas institucionales y a la vez la clasificación de las mismas. Dentro de esta misma línea, Dsntowski et al. (2018) determinaron que las instituciones de educación superior desarrollan acciones socialmente responsables que pueden representarse a través de 7 diferentes modelos de estrategia universitaria, los cuales reflejan las diferencias entre las universidades en términos de sus enfoques estratégicos generales. Miotto et al. (2018) plantean que para aumentar su legitimidad ante la comunidad las universidades deben incluir aspectos sociales y temas relacionados con la responsabilidad social en de su modelo estratégico.

\section{- Perspectiva 3: conexión RSU y sociedad}

Esta perspectiva representa el $5 \%$ del total de artículos de la red y está compuesta por investigaciones que plantean la relación y responsabilidad de las instituciones de educación superior con el desarrollo y cambio social.

A continuación, se exponen algunas apreciaciones de las publicaciones que hacen parte de esta perspectiva: el desarrollo de las comunidades locales, regionales y nacionales debe ser un elemento misional para las universidades y un proceso bidireccional que genere beneficio para la sociedad como para la universidad (Păunescu, Drăgan Gilmeanu y Găucă, 2017), lo anterior implica reconsiderar el papel de estas instituciones en la sociedad y evaluar la forma en que estas se relacionan con sus grupos de interés, además, repensar su papel como socios industriales y regionales (Jongbloed, Enders y Salerno, 2008).

En la actualidad, la sociedad se enfrenta a desafíos sin precedentes y cada vez más urgentes asociados con la aceleración del cambio ambiental, la escasez de recursos, el aumento de la desigualdad y la injusticia, bajo este nuevo panorama las universidades son consideras un agente de cambio con un papel protagónico (Stephens, Hernandez, Román, Graham y Scholz, 2008); bajo esta misma línea, la UNESCO (2009) en la Conferencia Mundial sobre la Educación Superior manifiesta "ante la complejidad de los desafíos

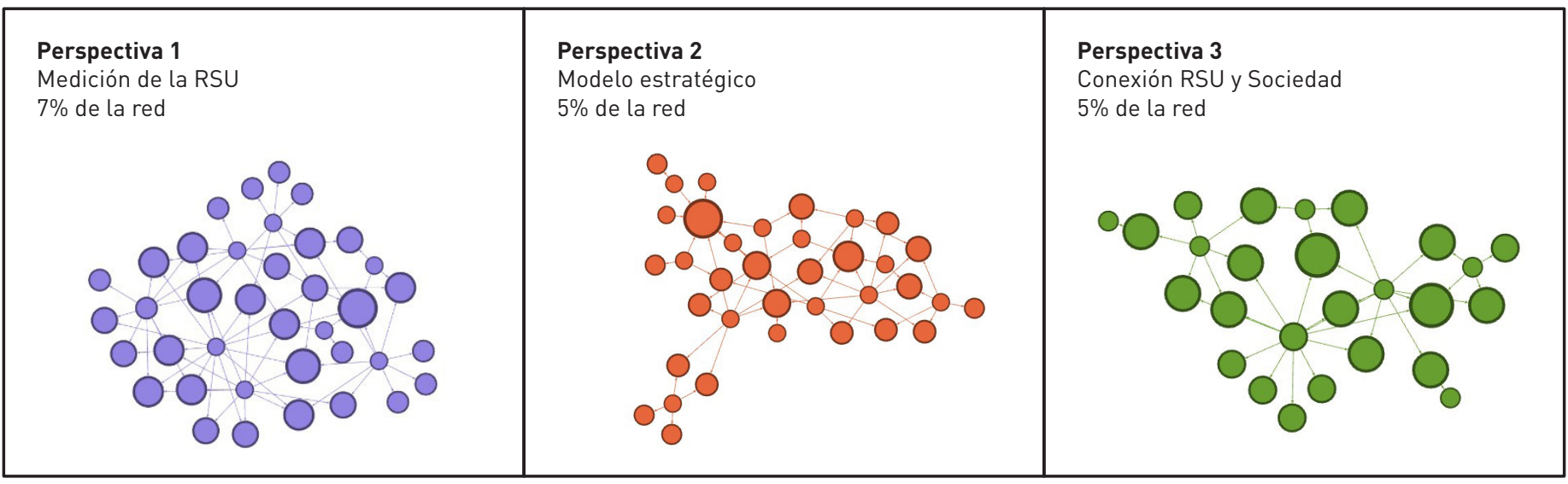

Figura 8. Perspectivas en RSU

Fuente: elaboración propia. 
mundiales, presentes y futuros, la educación superior tiene la responsabilidad social de hacer avanzar nuestra comprensión de problemas polifacéticos con dimensiones sociales, económicas, científicas y culturales, así como nuestra capacidad de hacerles frente" (p. 1).

\section{Conclusiones}

Se observa el crecimiento en relación con el número de publicaciones sobre RSU en los últimos años, aunque en términos generales la producción académica se acelera de manera importante a partir del año 2015 , seis años después de la publicación del documento más referenciado en este campo “Responsabilidad Social Universitaria: manual de primeros pasos" (Vallaeys et al., 2009)

El país que encabeza la producción de trabajos en el área de la RSU es España, y este liderazgo se presenta en las dos bases de datos empleadas en esta investigación (WoS y Scopus), lo que muestra que teóricamente no hay sesgo respecto a las fuentes. En el top 10 de los países con mayor producción en este campo 7 de ellos son latinoamericanos, lo que representa que el $54 \%$ de la producción dentro de este top se generó en esa región.

Respecto a las bases de datos, la que presenta mayor producción en el área es Scopus con $58 \%$ de las publicaciones sobre el $42 \%$ de WoS; en referencia al número de publicaciones en revistas, dentro top 10 de estas se encuentran 7 indexadas en Scopus, lo que muestra una inclinación a publicar dentro de esta base de datos investigaciones que se refieren a la RSU. La revista con mayor número de publicaciones (12 en total) es Opción, ésta tiene como área principal las ciencias sociales, artes y humanidades, el mayor número de sus publicaciones están relacionadas al campo de la educación ( $41 \%$ en total), también cabe destacar las revistas World review of entrepreneurship management and sustainable development y Revista digital de investigación en docencia universitaria RIDU, la primera cuenta con 9 artículos y se encuentra indexada en Scopus, la segunda cuenta con el mismo número de artículos y se encuentra indexada en WoS.

Esta área de estudio es relativamente joven, al verificar los documentos hegemónicos se evidencia que no superan los 15 años desde su publicación, además, solo se encontraron 200 publicaciones asociadas a este tema en las dos bases de datos más grandes e importantes del mundo. Sin lugar a dudas el autor protagónico es François Vallaeys, quien es el investigador más citado dentro de la categoría de Responsabilidad Social Universitaria en Google Scholar (2.045 citaciones en total), donde la red de cocitaciones y la red de RSU así lo corroboran.

Aun no hay claridad respecto a si las prácticas de RSU tienen algún impacto frente a sus grupos de interés, particularmente en temas como la imagen y la reputación, las investigaciones son en cierta medida contradictoras, por un lado Chile y Black (2015) determinaron que el compromiso de la universidad con la comunidad len términos de proyección sociall generó beneficios en términos de reconocimiento de marca y reputaciones para la institución; por otro lado, Plungpongpan et al. (2016) indican que para los estudiantes las acciones de RSU no son un factor decisivo para elegir una universidad u otra, lo que demuestra que es necesario dar mayor profundidad al tema con el fin de aportar al establecimiento de un consenso en estos términos. Adicionalmente, Latif (2017) sugiere llevar a cabo más investigaciones que permitan la construcción de una escala confiable para la medición de la RSU, pues es claro que el tema requiere una mayor investigación, en poblaciones diferentes con orígenes culturales diferentes, para así lograr una generalización de los conceptos.

La red presenta de manera preliminar tres perspectivas: perspectiva 1 (medición de la RSU), perspectiva 2 (estratégico) y perspectiva 3 (RSU y sociedad); sin embargo, no pueden considerarse definitivos ni concluyentes, ya que al ser un campo del conocimiento tan reciente las perspectivas no están integradas por un número importante de investigaciones que las consoliden, pero son una propuesta que invita a un análisis más exhaustivo.

Finalmente, según la literatura revisada, esta es la primera revisión sistemática sobre RSU que ha aplicado la teoría de grafos para analizar la producción científica en este campo. Este análisis ha permitido la visualización de una red que describe la interacción de 2.821 publicaciones durante un periodo de tiempo amplio, permitiendo identificar las publicaciones clásicas, estructurales y recientes, donde las últimas son de gran relevancia ya que permiten identificar las perspectivas o líneas de estudio en este campo; convirtiéndose en una guía para la comprensión de la evolución y estado actual de la RSU, un área de investigación joven, pero con un crecimiento importante.

Al igual que en cualquier tipo de investigación, la revisión presentada en este artículo muestra ciertas limitaciones. En primer lugar, la búsqueda inicial se realizó en las bases de datos WoS y Scopus, como consecuencia natural de esta situación, las investigaciones que no se incluyen en estas bases de datos están fuera del alcance del estudio actual. En segundo lugar, el tema de búsqueda University Social Responsibility podría implicar ciertas limitaciones, ya que puede excluir palabras clave relacionadas a la RSU. Para futuras investigaciones se sugiere realizar un metaanálisis de este campo, además, de profundizar en el análisis de las perspectivas propuestas.

\section{Conflictos de intereses}

Los autores declaran no tener ningún conflicto de intereses.

\section{Fuente de financiación}

Este artículo hace parte del proyecto de investigación titulado "Responsabilidad social y marketing social en el sistema universitario de Manizales", financiado por la universidad Católica Luis Amigó (Colombia). 


\section{Bibliografía}

Addor, N. y Melsen, L. A. (2019). Legacy, Rather Than Adequacy, Drives the Selection of Hydrological Models. Water Resources Research, 55(1), 378390. https://doi.org/10.1029/2018WR022958

Aldeanueva, I. y Arrabal, G. (2018). La comunicación y medición de la Responsabilidad Social Universitaria: Redes sociales y propuesta de indicadores. Revista Digital de Investigación en Docencia Universitaria, 12(1), 121-136. https://doi.org/10.19083/ridu.12.626

Almeida, F. y de Paula, L. G. (2019). The Place of Uncertainty in Heterodox Economics Journals: A Bibliometric Study. Journal of Economic Issues, 53(2), 553-562. https://doi.org/10.1080/00213624.2019.1603771

Alonso, J., Castiello, C. y Mencar, C. (2018). A Bibliometric Analysis of the Explainable Artificial Intelligence Research Field. Springer, 855, 3-15. https://doi.org/10.1007/978-3-319-91479-4

Aria, M. y Cuccurullo, C. (2017). bibliometrix: An R-tool for comprehensive science mapping analysis. Journal of Informetrics, 11(4), 959-975. https:// doi.org/10.1016/j.joi.2017.08.007

Aristimuño, M. y Rodriguez Monroy, C. (2014). Responsabilidad Social Universitaria. Su gestión desde la perspectiva de directivos y docentes. Estudio de caso: una pequeña universidad Latinoamericana. Interciencia, 39(6), 375-383.

Atakan, M. G. S. y Eker, T. (2007). Corporate identity of a socially responsible university - A case from the Turkish higher education sector. Journal of Business Ethics, 76(1), 55-68. https://doi.org/10.1007/s10551-006-9274-3

Bar-Ilan, J. (2008). Which h-index? - A comparison of WoS, Scopus and Google Scholar. Scientometrics, 74(2), 257-271. https://doi.org/10.1007/ s11192-008-0216-y

Bar-Ilan, J. (2010). Citations to the "Introduction to informetrics" indexed by WOS, Scopus and Google Scholar. Scientometrics, 82(3), 495-506. https:// doi.org/10.1007/s11192-010-0185-9

Bastian, M., Heymann, S. y Jacomy, M. (2009). Gephi: An Open Source Software for Exploring and Manipulating Networks. International AAAI Conference on Weblogs and Social Media.

Blondel, V. D., Guillaume, J. L., Lambiotte, R. y Lefebvre, E. (2008). Fast unfolding of communities in large networks. Journal of Statistical Mechanics: Theory and Experiment, 10(1), 2-11. https://doi. org/10.1088/1742-5468/2008/10/P10008

Bollobás, B. (1984). The Evolution of Random Graphs. American Mathematical Society, 286(1), 257-274. https://doi.org/10.2307/1999405

Bond, M. y Buntins, K. (2018). An analysis of the Australasian Journal of Educational Technology 2013-2017. Australasian Journal of Educational Technology, 34(4). https://doi.org/10.14742/ajet.4359

Bowen, H. R. (1960). Social Responsibilities of the Businessman. University of lowa Press. lowa: University of lowa Press. https://doi.org/10.2307/j. ctt20q1w8f

Bretón Lópéz, J. y Buela Casal, G. (2003). Análisis comparativo de las revistas de psicología clínica editadas en castellano. Revista mexicana de psicología, 20(1), 141-155.

Brivio Borja, A. R. y Montano Rodríguez, F. (2016). La responsabilidad social universitaria. Tratamiento en la UMET. Conrado, 12(1), 111-118.

Carroll. (1991). The Pyramid of Corporate Social Responsibility. Business Horizons, 34(4), 39-48. https://doi.org/10.1016/0007-6813(91)90005-G

Carroll. (1999). Corporate Social Responsibility: Evolution of a Definitional Construct. Business \& Society, 38(3), 268-295. https://doi. org/10.1177/000765039903800303

Carroll, A. (1979). A Three-Dimensional Conceptual Model of Corporate Performance. Academy of Management Review, 4(4), 497-505. https://doi. org/10.5465/amr.1979.4498296

Cervantes, O., Botero, C. M. y Finkl, C. W. (2018). State-of-the-Art Beach Ecosystem Management from the Tree of Science Platform. En C. M. Botero, 0. Cervantes, \& C. W. Finkl (Eds.), Beach Management Tools Concepts, Methodologies and Case Studies (pp. 3-19). Cham: Springer International Publishing. https://doi.org/10.1007/978-3-319-58304-4_1

Chile, L. M. y Black, X. M. (2015). University-community engagement: Case study of university social responsibility. Education, Citizenship and Social Justice, 10(3), 234-253. https://doi.org/10.1177/1746197915607278

De Bakker, F. G. A., Groenewegen, P. y Den Hond, F. (2005). A bibliometric analysis of 30 years of research and theory on corporate social responsibility and corporate social performance. Business and Society, 44(3), 283-317. https://doi.org/10.1177/0007650305278086
De la Calle, C. y Giménez, P. (2011). Aproximación al concepto de Responsabilidad Social del Universitario. Comunicación y Hombre, 7 , 237-247.

Díez, D. A. (2018). Virtudes públicasy toma de decisiones éticas en organizaciones estatales. La fusión UNE-Millicom en Colombia. Universidad Nacional de Colombia. Universidad Nacional de Colombia. Recuperado 15 febrero 2019, de http://bdigital.unal.edu.co/65675/

Dsntowski, T., Brdulak, H., Jastrzebska, E. y Legutko-Kobus, P. (2018). University Social Responsibility Strategies. e-mentor, (5), 4-12.

Flórez Guzmán, M., Rubio-Rodriguez, G. A., Rodrad Cooperativ, M. S. y UniveCurvelo Hasssl, J. (2017). Responsabilidad Social Universitaria "Una aproximación desde la percepción de la colectividad académica". Hermes, 17(1), 80-103.

Freeman. (1977). A Set of Measures of Centrality Based on Betweenness. Sociometry, 40(1), 35-41. https://doi.org/10.2307/3033543

Freeman, R. E. y Mcvea, J. (1984). A Stakeholder Approach to Strategic Management. The Blackwell Handbook of Strategic Management, (Januaryl, 183-201. https://doi.org/10.1111/b.9780631218616.2006.00007.x

Gaete Quezada, R. A. (2011). La responsabilidad social universitaria como desafio para la gestión estratégica de la Educación Superior: el caso de España. Revista de educación, (355), 109-133. https://doi.org/10.1016/ S2214-109X(17)30308-X

Garde Sánchez, R., Rodríguez Bolívar, M. P. y López-Hernández, A. M. (2013). Online disclosure of university social responsibility: A comparative study of public and private US universities. Environmental Education Research, 19(6), 709-746. https://doi.org/10.1080/13504622.2012.749976

Gasca-Pliego, E. y Olvera-García, J. C. (2011). Construir ciudadanía desde las universidades, responsabilidad social universitaria y desafíos ante el siglo XXI. Convergencia, 18(56), 37-58.

Gavel, Y. y Iselid, L. (2008). Web of Science and Scopus: A journal title overlap study. Online Information Review, 32(1), 8-21. https://doi. org/10.1108/14684520810865958

Gómez, C. R. y Solana, V. H. (2005). La producción científica Latinoamericana y la ciencia mundial: una revisión bibliográfica (1989-2003). Revista Interamericana de Bibliotecología, 28(1), 43-61.

Gómez, L. M., Alvarado Naveira, Y. y Pujol Bernabel, A. (2018). Practicando la Responsabilidad Social Universitaria en el Caribe: Perspectivas de los públicos internos. Revista Digital de Investigación en Docencia Universitaria, 12(1), 96-118.

Henríque Fuentes, G. R., Vallaeys, F. y Castrillón Garzón, M. A. (2018). El aprendizaje organizacional como herramienta para la universidad que aprende a ser responsable socialmente. Pensamiento Americano, 11(July), 116-140. https://doi.org/10.21803\%2Fpenamer.11.20.499

Hernández-Leal, E. J., Duque-Méndez, N. D. y Moreno-Cadavid, J. (2017). Big Data: una exploración de investigaciones, tecnologías y casos de aplicación. TecnoLógicas, 20(39), 15-38. https://doi. org/10.22430/22565337.685

Hirsch, J. E. (2005). An index to quantify an individual's scientific research output. PNAS, 102(46), 16569-16572. https://doi.org/10.1073/ pnas. 0507655102

Jongbloed, B., Enders, J. y Salerno, C. (2008). Higher education and its communities: Interconnections, interdependencies and a research agenda. Higher Education, 56(3), 303-324. https://doi.org/10.1007/ s10734-008-9128-2

Larrán-Jorge, M. y Andrades-Peña, F. J. (2017). Analysing the literature on university social responsibility: A review of selected higher education journals. Higher Education Quarterly, 71(4), 302-319. https://doi. org/10.1111/hequ.12122

Latif, K. F. (2017). The Development and Validation of Stakeholder-Based Scale for Measuring University Social Responsibility (USR). Social Indicators Research (Vol. 140). Springer Netherlands. https://doi.org/10.1007/ s11205-017-1794-y

López-Noriega, M. D., Zelthen-Hernández, L. y Carillo-Marín, A. M. (2016). La gestión en la universidad, una perspectiva de sus grupos de interés internos. Ra Ximhai, 12(6), 215-225.

Martí Noguera, J. J. (2016). El desarrollo regional como responsabilidad social de la universidad. Caso del ecoturismo en la provincia de Tungurahua. Revista Colombiana de Ciencias Sociales, 7(1), 96-113.

Martí Noguera, J. J., Martí-Vilar, M. y Almerich, G. (2014). Responsabilidad social universitaria: Influencia de valores yempatía en la autoatribución de comportamientos socialmente responsables. Revista Latinoamericana de Psicologia, 46(3), 160-168. https://doi.org/10.1016/S0120-0534(14)70019-6 
Martínez Martín, M. (2010). Educación y ciudadanía en sociedades democráticas: hacia una ciudadanía colaborativa. En Educación, valores y autonomía (Organizaci). Madrid: OEl.

Mazzetti, M. S. (2014). Universitaria, Analizando los constructos: Responsabilidad Social Universitaria y Compromiso Social. Mar del Plata.

Miotto, G., Blanco González, A. y Del Castillo Feito, C. (2018). Social Responsibility: A Tool for Legitimation in Spanish Universities' Strategic Plans. Trípodos, 42(42), 59-79.

Moravcsik, M. J. (1989). ¿Como evaluar la Ciencia y a los científicos? Revista española de documentación científica, 12(3), 313-323

Naranjo Africano, G. y Mejia Reatiga, C. (2018). La transferencia tecnológica como práctica de responsabilidad social universitaria. ArborCiencia Pensamiento Y Cultura, 194(789). https://doi.org/10.3989/ arbor.2018.789n3014

Ni, C., Sugimoto, C. y Jiang, J. (2011). Degree, Closeness, and Betweenness: Application of group centrality measurements to explore macrodisciplinary evolution diachronically. Proceedings of Issi 2011: The 13th Conference of the International Society for Scientometrics and Informetrics., 1, 605-616.

Novillo Díaz, L. A. (2019). La inclusion de grupos de atencion prioritaria en proyectos de vinculación de la sociedad. Conrado, 15(67), 75-80.

Osorio, N. y Zambrano, C. (2015). Discursos representacionales sobre transferencia social del conocimiento en la universidad de Carabobo. ORBIS, 32, 44-58.

Pacenza, M. I. y Silva P., Y. (2013). Análisis bibliométrico sobre responsabilidad social universitaria. Psychology, Society, \& Education, 5(2), 125-138. https://doi.org/10.25115/psye.v5i2.500

Păunescu, C., Drăgan Gilmeanu, D. y Găucă, O. (2017). Examining obligations to society for QS stars best ranked universities in social responsibility. Management and Marketing, 12(4), 551-570. https://doi.org/10.1515/ mmcks-2017-0033

Plungpongpan, J., Tiangsoongnern, L. y Speece, M. (2016). University social responsibility and brand image of private universities in Bangkok. Facilities, 30(4), 925-945. https://doi.org/10.1108/EL-01-2014-0022

Porter, M. E. y Kramer, M. R. (2006). Estrategia y sociedad. HBR Latinamerica, (December), 1-14. Recuperado el 15 febrero 2019, de: http://www.fundacionseres.org/Lists/Informes/Attachments/12/ Estrategia y Sociedad.pdf

Robledo, S., Osorio, G. y López, C. (2014). Networking en pequeña empresa: una revisión bibliográfica utilizando la teoria de grafos. Revista Vinculos, 11(2), 6-16. https://doi.org/10.14483/2322939X.9664

Sancho, R. (1990). Indicadores bibliométricos utilizados en la evaluación de la ciencia y la Tecnología. Revisión Bibliográfica. Revista española de documentación científica, 13(3-4), 842-865.

Sci2 Team. (2009). Science of Science (Sci2) Tool.

Stephens, J. C., Hernandez, M. E., Román, M., Graham, A. C. y Scholz, R. W. (2008). Higher education as a change agent for sustainability in different cultures and contexts. International Journal of Sustainability in Higher Education, 9(3), 317-338. https://doi.org/10.1108/14676370810885916

Toro, J. y del Rodríguez, M. P. (2017). Formación en Ética en las Organizaciones: Revisión de la Literatura Ethics Training in Organizations: Literature Review. Información Tecnológica, 28(2), 167-180. https://doi.org/10.4067/ S0718-07642017000200018

Trujillo Salazar, D. (2017). Mobile commerce: una revisión bibliográfica. NOVUM, Resvista de ciencias sociales aplicadas, 7, 59-77.

UNESCO. (2009). Conferencia Mundial sobre la Educación Superior-2009: La nueva dinámica de la educación superior y la investigación para el cambio social y el desarrollo. UNESCO.

Vallaeys. (2007). Breve Marco Teórico de RSU. Ética y RSU: Reflexión crítica en torno a la ética aplicada a la Responsabilidad Social Universitaria. ¿Nombre del cambio o gran cambio de nombre? La gestión ética e inteligente de los impactos universitarios, 1-10.

Vallaeys. (2014). La Responsabilidad Social Universitaria: un nuevo modelo universitario contra la mercantilización. Revista Iberoamericana de Educación Superior, 5, 105-117. https://doi.org/10.22201/ iisue.20072872e.2014.12.112

Vallaeys, F. (2004). Enseñar la ética y el desarrollo en la Universidad en la era planetaria. Maestría en gerencia social de la Pontificio Universidad Católica del Perú, 1-4. Recuperado el 15 febrero 2019, de: http://www.cyta.com. ar/biblioteca/bddoc/bdlibros/rse/334_as_ensenar_etica.pdf
Vallaeys, F. (2013). Responsabilidad Social Universitaria: una nueva filosofía de gestión ética e inteligente para las universidades. Educación superior y sociedad, 2(1), 177-206.

Vallaeys, F. (2018). Las diez falacias de la Responsabilidad Social Universitaria. Revista Digital de Investigación en Docencia Universitaria, 12, 34-58. https://doi.org/10.19083/ridu.12.716

Vallaeys, F. y Álvarez Rodríguez, J. (2019). Hacia una definición latinoamericana de Responsabilidad Social Universitaria. Aproximación a las preferencias conceptuales de los universitarios. Educación XX1, 22(1), 93-116. https://doi.org/10.5944/educxx1.19442

Vallaeys, F., De la Cruz, C. y Sasia, P. M. (2009). Responsabilidad Social Universitaria: Manual de primeros pasos. México, D.F.: McGrawHill Interamericana.

Vasilescu, R., Barna, C., Epure, M. y Baicu, C. (2010). Developing university social responsibility: A model for the challenges of the new civil society. Procedia - Social and Behavioral Sciences, 2(2), 4177-4182. https://doi. org/10.1016/j.sbspro.2010.03.660

Vázquez, J., Lanero, A. y Aza, C. (2015). Students experiences of university social responsability and perceptions of satisfaction and quality of service. Ekonomski Vjesnik, 28, 25-36.

Vieira, E. S. y Gomes, J. A. N. F. (2009). A comparison of Scopus and Web of science for a typical university. Scientometrics, 81(2), 587-600. https:// doi.org/10.1007/s11192-009-2178-0

Wallis, W. D. (2007). A Beginner's Guide to Graph Theory (2nd Ed.). Boston: Birkhäuser Boston. http://link.springer.com/10.1007/978-1-4757-3134-7

Wasserman, S. y Faust, K. (1994). Social Network Analysis: Methods and applications. Cambridge: Cambridge University Press.

Zuluaga, M., Robledo, S., Osorio-Zuluaga, G. A., Yathe, L., Gonzalez, D. y Taborda, G. (2016). Metabolómica y Pesticidas: Revisión sistemática de literatura usando teoría de grafos para el análisis de referencias. Nova, 13(25), 121-138. https://doi.org/10.22490/24629448.1735 